\title{
Evaluación ultrasonográfica de las medidas dorsales y del anca y su relación con metabolitos lipídicos en ganado Brahman
}

\section{Ultrasonographic evaluation of back and hip measurements and their relationship to lipid profile in Brahman cattle}

\author{
Néstor Alonso Villa A. ${ }^{1 *}$ M.Sc, Paulo Duque $M,{ }^{1}$ MVZ Ariel Jiménez $R,{ }^{2}$ M.Sc, \\ Alejandro Ceballos M, ${ }^{1} \mathrm{Ph} . \mathrm{D}$.
}

\begin{abstract}
1Universidad de Caldas, Programa de Medicina Veterinaria y Zootecnia, Departamento de Sistemas de Producción, A.A 275 Manizales, Colombia. Grupo de Investigación en Biología de la Producción Pecuaria. ${ }^{2}$ Asociación Colombiana de Criadores de Ganado Cebú (ASOCEBÚ). ${ }^{*}$ Correspondencia: navilla@ucaldas.edu.co
\end{abstract}

Recibido: Marzo de 2011; Aceptado: Julio de 2012.

\section{RESUMEN}

Objetivo. Determinar las medidas ultrasonográficas de musculatura y grasa en ganado Brahman estabulado y su asociación con edad, sexo y los valores sanguíneos de colesterol total (CT), lipoproteínas de alta densidad (HDL) y baja densidad (LDL), triacilgliceroles (TG), $\beta$-hidroxibutiratos $(\beta-O H B)$ y tiroxina libre $\left(\mathrm{T}_{4}\right)$. Material y métodos. Se seleccionaron 180 animales entre machos y hembras con edades entre 9 y 24 meses. De cada animal se tomaron medidas ultrasonográficas del área de ojo del lomo (AOL), profundidad del músculo glúteo medio (PMGM), espesor de grasa dorsal (EGD) y espesor de grasa del anca (EGA), y muestra de sangre para determinar los metabolitos sanguíneos. Se emplearon correlaciones y regresión lineal para establecer las asociaciones entre las variables. Resultados. El sexo y el peso del animal fueron predictores útiles de las medidas de la musculatura y cobertura grasa en el dorso y el anca del animal. Igualmente, el sexo y el peso produjeron cambios en la concentración de los metabolitos séricos estudiados. La correlación entre algunos metabolitos séricos y EGD, PMGM, y EGA fue baja. Conclusiones. La relación que existe entre las evaluaciones de la musculatura y la cobertura grasa es baja, lo que indica que los metabolitos séricos evaluados no son predictores adecuados del desempeño productivo de ganado Brahman para exposición mantenido en estabulación.

Palabras clave: Colesterol, grasas, lípidos, músculos (Fuente:CAB). 


\begin{abstract}
Objective. To establish the relationship between ultrasonographic measurements of muscle and fat in back and hip of Brahman cattle fed for cattle shows and their association with serum levels of total cholesterol (CT), high density lipoprotein (HDL), low density lipoprotein (LDL), triglyceride (TG), $\beta$-hydroxybutirate ( $\beta$-OHB), and free thyroxin (T4). Materials and methods. One hundred and eighty animals were purposively selected. The age varied between 9 and 24 months. Ultrasonographic measurements from the loin eye area (AOL), depth of gluteus medius (PMGM), backfat thickness (EGD), and hip fat thickness (EGA) from each animal were recorded. Blood serum samples were collected to determine total cholesterol (CT), lipoproteins (HDL and LDL), triglycerides (TG), and thyroxin (T4). Correlation and linear regression analyses were conducted. Results. Gender and weight were related to muscle and fat coverage measurements in the backs and hips of the animals. Differences in serum metabolites were also related to gender and weight. Although there was a significant correlation between the ultrasonographic measurements and serum metabolites, the associations were weak. Conclusions. The relationship between ultrasonographic measurements of back and hip with serum lipid metabolites is weak, which indicates that those metabolites are not suitable to predict the productive performance of Brahman cattle fed for cattle shows.
\end{abstract}

Key words: Cholesterol, fat, lipids, muscles (Source:CAB).

\section{INTRODUCCIÓN}

En los sistemas productivos donde se crían animales cebuínos para exposiciones ganaderas, la suplementación alimenticia comienza desde edades tempranas, utilizándose generalmente alimentos balanceados. La composición muscular es un indicador del balance nutricional durante el crecimiento de los bovinos destinados a la producción de carne y es sabido que el exceso en la suplementación alimenticia produce una mayor acumulación de grasa subcutánea e intramuscular y una mayor síntesis de proteína (1).

Las consecuencias de la sobrealimentación en machos son la obesidad y problemas de aplomos, los cuales pueden contribuir en forma indirecta a alteraciones de la conducta sexual (2). El sobrepeso en la hembra, entendido como un evidente incremento de la condición corporal (sobreacondicionamiento), está relacionado con cambios en el perfil lipídico a nivel sanguíneo y ovárico induciendo alteraciones de la fertilidad (3).

La ultrasonografía se ha propuesto como un método in vivo para evaluar el grado de engrasamiento y el desarrollo muscular en bovinos, siendo un método no invasivo y de fácil interpretación (4). En ganado Brahman, si bien se ha utilizado la ultrasonografía para evaluar el grado de engrasamiento, no se conocen antecedentes acerca de la asociación entre las medidas ultrasonográficas AOL, PMGM, EGD y EGA y los indicadores metabólicos del balance de energía.

Se ha propuesto como hipótesis para este estudio que aquellos animales más pesados y con medidas ultrasonográficas mayores para las variables descritas, tendrían cambios en los metabolitos sanguíneos relacionados con el metabolismo energético. En consideración a lo anterior, el objetivo de este estudio fue conocer las medidas ultrasonográficas de grasa y musculatura en lomo y anca en ganado Brahman estabulado para exposición y su asociación con el sexo, la edad y el peso del animal, y con los valores sanguíneos para metabolitos relacionados con el metabolismo energético como CT, HDL, LDL, TG, $\beta-O H B$ y $\mathrm{T}_{4}$.

\section{MATERIAL Y MÉTODOS}

Animales. Se seleccionaron aleatoriamente de un listado de animales Brahman estabulados para exposición y registrados en la asociación de criadores, 180 individuos (i.e. 96 hembras y 84 machos) clínicamente sanos, con edades entre 9 y 24 meses, que fueron pesados al momento de llegar a los eventos y exhibidos en la Feria Nacional Cebú 2007 y en la Feria Internacional Cebú 2008.

Datos ultrasonográficos. Se llevó a cabo la medición del AOL, PMGM, EGD tomada al nivel de las costillas 12 y 13 , y EGA para cada uno de los animales (5). Para las mediciones, previa sujeción del animal, se utilizó un ecógrafo ESAOTE PIE MEDICAL ${ }^{\circledR}$ modelo Aquila con una sonda sectorial de $3.5 \mathrm{MHz}$ (Pie Medical, Philipsweg, Holanda) y fueron realizadas por un técnico siguiendo la metodología previamente descrita (5). El AOL se expresó en $\mathrm{cm}^{2}$ y la PMGM en $\mathrm{cm}$; el espesor de las grasas dorsal y del anca en $\mathrm{mm}$. 
Toma de muestras y análisis. De cada animal se tomó una muestra de sangre $(10 \mathrm{~mL})$ mediante venopunción coccígea depositándose en tubos al vacío (Vacutainer, Becton-Dickinson, Franklin Lakes, NJ, USA) sin anticoagulante. Las muestras fueron centrifugadas a $1800 \mathrm{~g}$ por 5 minutos y se refrigeraron a $4^{\circ} \mathrm{C}$ hasta su envío al laboratorio donde se congelaron a $-20^{\circ} \mathrm{C}$ hasta su análisis.

Se determinó la concentración de $\mathrm{CT}$, $\mathrm{HDL}$, $\mathrm{LDL}, \mathrm{TG}, \beta-\mathrm{OHB}$ y $\mathrm{T}_{4}$ mediante la utilización de reactivos comerciales (BioSystems, Barcelona, España) en el Laboratorio de Patología Clínica del Hospital Veterinario de la Universidad de Caldas.

Análisis estadístico. Se llevaron a cabo análisis separados para evaluar la asociación incondicional entre las variables explicatorias (peso, sexo y edad del animal) y las variables respuesta (AOL, PMGM, EGD y EGA). Igualmente, se evaluó separadamente el efecto del peso, sexo y edad sobre los indicadores del metabolismo lipídico. Pese a lo anterior, algunas de las variables explicatorias fueron consideradas como variables respuesta, como por ejemplo en la evaluación de la asociación entre la edad y el sexo, y entre el peso y el sexo. Estas asociaciones se establecieron mediante correlación simple y regresión lineal simple (6). Las variables que estaban incondicionalmente asociadas $(p \leq 0.10)$ fueron retenidas para la construcción de un modelo de regresión lineal multivariado, que fue posteriormente reducido mediante la selección de paso atrás (backward selection), dejando en el modelo final las variables que presentaban un valor $p \leq 0.05(6)$.
Todos los análisis incluyeron las variables independientes: peso del animal $(\mathrm{kg})$, sexo (hembra o macho) y edad categorizada como 0 (9 a 11 meses), 1 (12 a 14 meses), 2 (15 a 17 meses), 3 (18 a 20 meses) y 4 (21 a 23 meses). El sexo y el peso de los animales fueron evaluados como potenciales variables de confusión en las diferentes asociaciones analizadas. Los supuestos del modelo fueron evaluados mediante el análisis de los residuos, y las asociaciones significativas $(p \leq 0.05)$ fueron presentadas como el promedio del cuadrado mínimo basado en el modelo. Los análisis fueron realizados usando los comandos 'pwcorr' y 'reg' en Stata version 12.1 (StataCorp, College Station, TX, USA).

\section{RESULTADOS}

La edad promedio de los animales del estudio fue $458 \pm 122$ días, con un rango entre 277 y 716 días, no observándose diferencias según el sexo $(P=0,87)$. El peso promedio fue $517 \pm 117 \mathrm{~kg}$, con un peso mínimo de $311 \mathrm{~kg}$ y un máximo de $818 \mathrm{~kg}$. Los machos fueron $78.9 \pm 16.4 \mathrm{~kg}$ más pesados que las hembras $(p<0.001)$.

El promedio y la desviación estándar para cada una de las variables analizadas según la edad y el sexo se presenta en las tablas 1 y 2 . En algunas de las variables estudiadas, como por ejemplo EGD y TG, se observó una alta variación en los datos; mientras que para otras variables la variación fue menor, como en el caso de PMGM y $\mathrm{T}_{4}$.

Tabla 1. Promedio $(X)$ y desviación estándar (DE) para las medidas ultrasonográficas según el sexo y la edad en ganado Brahman estabulado.

\begin{tabular}{|c|c|c|c|c|c|c|c|c|c|c|}
\hline & \multicolumn{2}{|c|}{$\mathbf{n}$} & \multicolumn{2}{|c|}{ AOL $\left(\mathrm{cm}^{2}\right)$} & \multicolumn{2}{|c|}{ PMGM (cm) } & \multicolumn{2}{|c|}{ EGD (mm) } & \multicolumn{2}{|c|}{ EGA (mm) } \\
\hline & $\mathbf{H}^{2}$ & M & $\mathbf{H}$ & $\mathbf{M}$ & $\mathbf{H}$ & M & $\mathbf{H}$ & M & $\mathbf{H}$ & M \\
\hline $0^{1}$ & 37 & 31 & $79 \pm 10.2$ & $89 \pm 14.0$ & $7.9 \pm 0.7$ & $8.6 \pm 0.8$ & $7.5 \pm 4.1$ & $5.5 \pm 2.1$ & $11.6 \pm 3.8$ & $9.3 \pm 2.9$ \\
\hline 1 & 21 & 22 & $90 \pm 12.8$ & $98 \pm 22.9$ & $8.8 \pm 1.0$ & $9.6 \pm 0.8$ & $12.5 \pm 5.5$ & $9.0 \pm 2.4$ & $16.6 \pm 4.7$ & $12.6 \pm 4.6$ \\
\hline 2 & 19 & 17 & $103 \pm 7.8$ & $116 \pm 10.5$ & $9.2 \pm 0.9$ & $10.2 \pm 0.9$ & $15.8 \pm 3.6$ & $13.2 \pm 5.3$ & $19.4 \pm 4.2$ & $15.9 \pm 5.2$ \\
\hline 3 & 12 & 8 & $115 \pm 6.8$ & $122 \pm 8.9$ & $9.7 \pm 1.2$ & $10.8 \pm 1.2$ & $24.3 \pm 7.2$ & $11.9 \pm 5.8$ & $27.4 \pm 7.5$ & $17.4 \pm 5.7$ \\
\hline 4 & 7 & 6 & $110 \pm 8.1$ & $111 \pm 10.0$ & $9.3 \pm 1.1$ & $11.0 \pm 0.5$ & $23.1 \pm 5.0$ & $15.2 \pm 6.2$ & $28.3 \pm 5.5$ & $20.0 \pm 5.5$ \\
\hline
\end{tabular}

${ }^{1}$ Categorías de edad: 0 (9 a 11 meses), 1 (12 a 14 meses), 2 (15 a 17 meses), 3 (18 a 20 meses) y 4 (21 a 23 meses)

${ }^{2} \mathrm{H}$ : Hembras. M: Machos

Tabla 2. Promedio $(X)$ y desviación estándar (DE) para metabolitos sanguíneos según el sexo y la edad en ganado Brahman estabulado.

\begin{tabular}{|c|c|c|c|c|c|c|c|c|c|c|c|c|c|c|}
\hline & \multicolumn{2}{|c|}{ n } & \multicolumn{2}{|c|}{$\begin{array}{c}\text { CT } \\
(\mathrm{mmol} / \mathrm{L})\end{array}$} & \multicolumn{2}{|c|}{$\begin{array}{c}\text { HDL } \\
(\mathrm{mmol} / \mathrm{L})\end{array}$} & \multicolumn{2}{|c|}{$\begin{array}{c}\text { LDL } \\
(\mathrm{mmol} / \mathrm{L})\end{array}$} & \multicolumn{2}{|c|}{$\begin{array}{c}\text { TG } \\
(\mathrm{mmol} / \mathrm{L})\end{array}$} & \multicolumn{2}{|c|}{$\begin{array}{c}\beta-O H B \\
(\mathrm{mmol} / \mathrm{L})\end{array}$} & \multicolumn{2}{|c|}{$\begin{array}{c}\mathrm{T}_{4} \\
(\mathrm{pg} / \mathrm{mL})\end{array}$} \\
\hline & $\mathbf{H}^{2}$ & M & $\mathbf{H}$ & $\mathbf{M}$ & $\mathbf{H}$ & M & $\mathbf{H}$ & M & H & M & $\mathbf{H}$ & M & H & $\mathbf{M}$ \\
\hline $0^{1}$ & 37 & 31 & $6.9 \pm 1.9$ & $5.9 \pm 1.5$ & $2.8 \pm 0.8$ & $2.4 \pm 0.6$ & $4.1 \pm 1.6$ & $3.4 \pm 1.2$ & $0.6 \pm 0.4$ & $0.4 \pm 0.3$ & $0.5 \pm 0.1$ & $0.7 \pm 0.2$ & $17.2 \pm 2.4$ & $17.4 \pm 2.6$ \\
\hline 1 & 21 & 22 & $7.8 \pm 1.4$ & $7.2 \pm 1.2$ & $3.0 \pm 0.7$ & $2.8 \pm 0.6$ & $4.8 \pm 0.9$ & $4.4 \pm 0.9$ & $0.6 \pm 0.4$ & $0.5 \pm 0.5$ & $0.5 \pm 0.2$ & $0.6 \pm 0.2$ & $18.0 \pm 1.5$ & $17.6 \pm 2.9$ \\
\hline 2 & 19 & 17 & $8.1 \pm 1.9$ & $6.7 \pm 1.2$ & $3.1 \pm 0.6$ & $2.6 \pm 0.6$ & $5.0 \pm 1.7$ & $4.1 \pm 1.2$ & $0.6 \pm 0.4$ & $0.3 \pm 0.3$ & $0.6 \pm 0.1$ & $0.5 \pm 0.1$ & $17.0 \pm 1.6$ & $18.1 \pm 2.1$ \\
\hline 3 & 12 & 8 & $6.8 \pm 1.5$ & $7.2 \pm 2.1$ & $2.7 \pm 0.5$ & $2.9 \pm 0.6$ & $4.0 \pm 1.2$ & $4.3 \pm 2.0$ & $0.7 \pm 0.4$ & $0.5 \pm 0.3$ & $0.6 \pm 0.1$ & $0.5 \pm 0.1$ & $17.4 \pm 3.3$ & $19.1 \pm 1.7$ \\
\hline 4 & 7 & 6 & $7.6 \pm 1.3$ & $7.3 \pm 1.8$ & $3.1 \pm 0.9$ & $3.0 \pm 0.4$ & $4.5 \pm 1.9$ & $4.2 \pm 1.4$ & $0.7 \pm 0.3$ & $0.6 \pm 0.4$ & $0.5 \pm 0.1$ & $0.6 \pm 0.3$ & $19.9 \pm 2.7$ & $17.7 \pm 1.5$ \\
\hline
\end{tabular}

${ }^{1}$ Categorías de edad: 0 (9 a 11 meses), 1 (12 a 14 meses), 2 (15 a 17 meses), 3 (18 a 20 meses) y 4 (21 a 23 meses)

${ }^{2} \mathrm{H}$ : Hembras. M: Machos 
La variable peso estaba relacionada en forma significativa con las variables sexo y edad; por consiguiente, estas variables fueron excluidas del modelo final en cuanto al análisis de su efecto sobre el AOL para evitar la colinearidad, dejando solamente como variable independiente el peso del animal. En cuanto a las otras variables dependientes, se optó por dejar en el modelo final el efecto del sexo siempre y cuando fuera un predictor significativo.

Medidas Ultrasonográficas. Según se mencionó anteriormente, el análisis de la asociación entre el sexo y la edad con las variables dependientes indicó que entre las variables peso y AOL había una colinearidad significativa. Lo anterior significa que estas variables estaban explicando la misma respuesta, por lo que fueron excluidas del modelo final dado que la utilidad de este modelo es predictiva y no buscando una asociación causal.

El peso del animal estaba asociado significativamente con las variables AOL, PMGM, EGD y EGA. En general se observó que la medida para estas variables aumenta en los machos y según el peso del animal (Tabla 3 ). Además, se observó una interacción significativa entre el sexo y el peso sobre el EGD y el EGA, lo que indica que el efecto del peso depende del sexo del animal.

Tabla 3. Modelo final para la evaluación de la asociación entre el peso y el sexo del animal con las medidas ultrasonográficas en ganado Brahman estabulado.

\begin{tabular}{|c|c|c|c|}
\hline Variable & $\begin{array}{c}\text { Coeficiente } \\
( \pm \mathrm{EE})\end{array}$ & $\mathbf{R}^{2}$ & $\mathbf{P}$ \\
\hline $\mathrm{AOL}\left(\mathrm{cm}^{2}\right)$ & & 0.57 & \\
\hline Intercepto & $34.9 \pm 4.2$ & & $<0.001$ \\
\hline Peso & $0.12 \pm 0.01$ & & $<0.001$ \\
\hline PMGM (cm) & & 0.49 & \\
\hline Intercepto & $5.5 \pm 0.3$ & & $<0.001$ \\
\hline Peso & $0.007 \pm 0.001$ & & $<0.001$ \\
\hline \multicolumn{4}{|l|}{ Sexo } \\
\hline Hembras & 0.0 & & \\
\hline Machos & $0.40 \pm 0.14$ & & 0.005 \\
\hline $\mathrm{EGD}(\mathrm{mm})$ & & 0.64 & \\
\hline Intercepto & $-16.59 \pm 2.08$ & & $<0.001$ \\
\hline Peso & $0.063 \pm 0.004$ & & $<0.001$ \\
\hline \multicolumn{4}{|l|}{ Sexo } \\
\hline Hembras & 0.0 & & \\
\hline Machos & $10.1 \pm 3.0$ & & 0.001 \\
\hline Interacción peso*sexo & $-0.03 \pm 0.01$ & & $<0.001$ \\
\hline EGA $(\mathrm{mm})$ & & 0.61 & \\
\hline Intercepto & $-12.16 \pm 2.21$ & & $<0.001$ \\
\hline Peso & $0.062 \pm 0.005$ & & $<0.001$ \\
\hline \multicolumn{4}{|l|}{ Sexo } \\
\hline Hembras & 0.00 & & \\
\hline Machos & $10.0 \pm 3.2$ & & 0.002 \\
\hline Interacción peso*sexo & $-0.03 \pm 0.01$ & & $<0.001$ \\
\hline
\end{tabular}

Con estos resultados, el EGD estimado para una hembra de $500 \mathrm{~kg}$ es $14.9 \pm 0.08 \mathrm{~mm}$, mientras que para un macho el valor estimado sería $10.0 \pm 2.1 \mathrm{~mm}$. Con respecto al EGA, en una hembra del mismo peso el valor estimado es $18.8 \pm 2.2 \mathrm{~mm}$, en un macho el valor sería $13.9 \pm 1.5 \mathrm{~mm}$.

Bioquímica sanguínea. Las relaciones encontradas entre las variables peso y sexo de los animales con los metabolitos sanguíneos se presentan en la tabla 4.

La concentración promedio de colesterol estuvo asociada con el peso y el sexo del animal, observándose que las hembras tuvieron una mayor concentración (Tabla 4). Con respecto a las lipoproteínas de alta y baja densidad se encontró una asociación significativa con el sexo, y al igual que para el colesterol, los valores más altos se encontraron en las hembras y los animales más pesados (Tabla 4). A diferencia de lo anterior, la concentración sérica de TG no estuvo asociada con el peso.

Al analizar la proporción de lipoproteínas con respecto a la concentración total de colesterol, se observó que la fracción HDL varió entre 38 y

Tabla 4. Modelo final para la asociación entre el peso y el sexo del animal con los indicadores del perfil lipídico en ganado Brahman estabulado.

\begin{tabular}{|c|c|c|c|c|}
\hline & Variable & Coeficiente ( \pm EE) & $\mathbf{R}^{2}$ & $\mathbf{P}$ \\
\hline \multirow[t]{6}{*}{ CT } & & & 0.09 & \\
\hline & Intercepto & $5.91 \pm 0.55$ & & $<0.001$ \\
\hline & Peso & $0.003 \pm 0.001$ & & 0.007 \\
\hline & Sexo & & & \\
\hline & Hembras & 0.0 & & \\
\hline & Machos & $-1.00 \pm 0.25$ & & $<0.001$ \\
\hline \multirow[t]{6}{*}{$\mathrm{HDL}$} & & & 0.06 & \\
\hline & Intercepto & $2.40 \pm 0.23$ & & $<0.001$ \\
\hline & Peso & $0.001 \pm 0.005$ & & 0.05 \\
\hline & Sexo & & & \\
\hline & Hembras & 0.0 & & \\
\hline & Machos & $-0.35 \pm 0.48$ & & 0.001 \\
\hline \multirow[t]{6}{*}{ LDL } & & & 0.06 & \\
\hline & Intercepto & $3.42 \pm 0.23$ & & $<0.001$ \\
\hline & Peso & $0.002 \pm 0.001$ & & 0.02 \\
\hline & Sexo & & & \\
\hline & Hembras & 0.0 & & \\
\hline & Machos & $-0.65 \pm 0.21$ & & 0.003 \\
\hline \multirow[t]{5}{*}{ TG } & & & 0.04 & \\
\hline & Intercepto & $0.59 \pm 0.38$ & & $<0.001$ \\
\hline & Sexo & & & \\
\hline & Hembras & 0.0 & & \\
\hline & Machos & $-0.14 \pm 0.05$ & & 0.008 \\
\hline \multirow[t]{3}{*}{$\mathrm{T}_{4}$} & & & 0.03 & \\
\hline & Intercepto & $15.83 \pm 0.81$ & & $<0.001$ \\
\hline & Peso & $0.003 \pm 0.001$ & & 0.02 \\
\hline
\end{tabular}


Tabla 5. Matriz de correlaciones entre las medidas de musculatura y grasa dorsal en el lomo y el anca y los metabolito sanguíneos en ganado Brahman para exposición y mantenido en estabulación.

\begin{tabular}{|c|c|c|c|c|c|c|c|c|c|}
\hline Variables & AOL & EGD & PMGM & EGA & CT & HDL & LDL & TG & В-Онв \\
\hline $\mathrm{AOL}$ & 1.000 & & & & & & & & \\
\hline EGD & 0.465 & 1.000 & & & & & & & \\
\hline $\mathrm{p}$ & 0.000 & & & & & & & & \\
\hline PMGM & 0.575 & 0.311 & 1.000 & & & & & & \\
\hline $\mathrm{p}$ & 0.000 & 0.000 & & & & & & & \\
\hline EGA & 0.410 & 0.812 & 0.209 & 1.000 & & & & & \\
\hline $\mathrm{p}$ & 0.000 & 0.000 & 0.005 & & & & & & \\
\hline Ст & 0.094 & 0.251 & 0.169 & 0.166 & 1.000 & & & & \\
\hline $\mathrm{p}$ & 0.209 & 0.001 & 0.024 & 0.026 & & & & & \\
\hline HDL & 0.045 & 0.182 & 0.090 & 0.130 & 0.586 & 1.000 & & & \\
\hline $\mathrm{p}$ & 0.551 & 0.015 & 0.232 & 0.085 & 0.000 & & & & \\
\hline LDL & 0.091 & 0.214 & 0.159 & 0.136 & 0.918 & 0.217 & 1.000 & & \\
\hline $\mathrm{p}$ & 0.226 & 0.004 & 0.035 & 0.070 & 0.000 & 0.003 & & & \\
\hline TG & -0.013 & 0.138 & -0.033 & 0.166 & 0.099 & 0.272 & -0.014 & 1.000 & \\
\hline $\mathrm{p}$ & 0.862 & 0.065 & 0.665 & 0.027 & 0.184 & 0.000 & 0.857 & & \\
\hline$\beta-\mathrm{OHB}$ & -0.076 & -0.077 & -0.074 & -0.022 & 0.014 & 0.089 & -0.027 & -0.098 & 1.000 \\
\hline $\mathrm{p}$ & 0.311 & 0.310 & 0.327 & 0.771 & 0.851 & 0.240 & 0.722 & 0.192 & \\
\hline
\end{tabular}

$41 \%$ tanto en las hembras como en los machos. Mientras que la fracción LDL varió entre 58 y $62 \%$ con respecto al colesterol total.

La concentración sérica de $\beta$-hidroxibutiratos no estuvo asociada con las variables sexo $(p=0.09)$ y peso del animal $(p=0.60)$.

Con respecto a la $\mathrm{T}_{4}$, ésta presentó una asociación significativa solamente con el peso del animal $(p=0.02$; Tabla 4).

No todas las variables estudiadas para evaluar la musculatura y la cobertura grasa en el dorso y el anca estaban relacionadas con los metabolitos sanguíneos. Aunque el CT y otros indicadores del metabolismo lipídico estaban relacionados con EGD, PMGM y EGA en forma significativa, los valores de correlación fueron bajos (Tabla 5).

\section{DISCUSIÓN}

El valor predictivo para la musculatura y la cobertura grasa en el dorso y el anca del animal, presentaron un coeficiente de determinación alto, lo que permite indicar que al menos un $50 \%$ de la variación en estas medidas estaba asociada con las variables explicatorias incluidas en el modelo (6). Por el contrario, la edad y el peso del animal explicaron una baja proporción de la variación en las variables bioquímicas estudiadas.

Los coeficientes de correlación observados para la evaluación entre la musculatura y la cobertura grasa en el dorso y el anca, fueron bajos, indicando una débil asociación lineal entre las variables (6).
Las medidas ultrasonográficas observadas fueron ligeramente más elevadas a lo descrito en otros estudios involucrando animales Brahman rojo y gris a los 18 meses de edad y mantenidos en pastoreo (1). Así como también fueron mayores a lo observado por otros autores (7), quienes hallaron un promedio para AOL de 45.2, 48.1 y $39.1 \mathrm{~cm}^{2}$ en animales con edades entre 9 y 12 meses. En animales cruzados de las razas Blanco Orejinegro x Cebú, Romosinuano x Cebú y Angus x Cebú con edades entre 18 y 24 meses, se ha encontrado un promedio menor para el AOL con respecto a los resultados obtenidos en este estudio (8). Estas diferencias pueden deberse a factores nutricionales, ya que los animales mantenidos en pastoreo podrían estar recibiendo una menor concentración de nutrientes comparado con animales estabulados como es el caso de los individuos observados en el presente estudio. Además, puede haber diferencias genéticas debidas a la raza o los cruzamientos entre ellas (9).

En este estudio se hallaron valores mayores de PMGM a los observados previamente en novillos Hereford de 16 meses de edad, donde encontraron un promedio de $7.8 \mathrm{~cm}$ (10). No obstante en otro estudio, reportan un promedio de $11.6 \mathrm{~cm}$ en toros jóvenes de varias razas (11). Estos mismos autores indicaron que las mediciones ultrasonográficas de animales vivos, tales como la PMGM y el EGA, permiten predecir más exactamente el porcentaje magro de las canales bovinas en comparación con las mediciones reales de la canal.

Para el EGD los resultados fueron mayores a los observados previamente para animales cruzados, 
observándose diferencias entre machos y hembras y según la edad (7). Ademas, estos mismos autores encontraron que los valores para el EGD aumentaban con la edad del animal y eran mayores en hembras, similar a lo obtenido en este estudio. En machos Brahman en crecimiento y mantenidos en pastoreo, los valores para EGD fueron más bajos que los obtenidos en este estudio (1). En general, los animales mantenidos bajo condiciones de pastoreo tienen un EGD inferior a los animales que son mantenidos bajo condiciones de estabulación, así como también hay diferencias según la raza y la zona geográfica de ubicación de las explotaciones (12).

El EGD en los animales de este estudio fue mayor a lo que se indica en la literatura, lo que está relacionado con una mayor y más rápida deposición de grasa dorsal que grasa intramuscular, lo que no es adecuado para obtener una canal de alta calidad y permitir una mejor comercialización (13). Se sabe que los animales más precoces en su desarrollo empiezan a fijar grasa a una edad más temprana, pues durante el crecimiento estos animales conforman primero la masa muscular y una vez que han alcanzado su crecimiento pico, se inicia el depósito de grasa (4). Este fenómeno unido a una sobrealimentación, característico de los programas de estabulación para ganado de exposición, estaría explicando la acumulación grasa desde una edad más temprana comparada con los animales mantenidos en pastoreo.

El EGA es una medida mejor valorada que el EGD, ya que el EGA puede ser mayor a la grasa del lomo de los animales debido a procesos fisiológicos; además, es la medida usada para determinar con mayor precisión el total de grasa corporal, ya que es altamente repetible y más fácil de evaluar e interpretar (8). Los resultados obtenidos en este trabajo concuerdan con otros estudios, donde la medición del EGA fue superior al EGD, lo que se encuentra relacionado con la distribución de la grasa de cobertura en el animal vivo, observándose además una alta correlación con el EGD (10). Los valores de EGA observados fueron similares a los descritos previamente para machos Hereford (10). No obstante, fueron mayores a los señalados para hembras en crecimiento Bos taurus (5).

Con respecto a los metabolitos sanguíneos, el CT fue superior a valores previamente descritos $(14,15)$, y a otros valores observados en vacas Brahman suplementadas y no suplementadas con grasas, cuyos valores fluctuaron entre 2.5 y $3.0 \mathrm{mmol} / \mathrm{L}$ (16). Se sabe que la concentración de CT está influenciada más por variables no relacionadas con el sexo, la edad o el peso del animal, que fueron los predictores considerados en este estudio. Entre otros factores, el CT puede estar influenciado por la raza y la pastura que consumen los animales como base de la alimentación (17).

La relación de HDL y LDL se encontró invertida con relación a lo descrito para bovinos (18) y para vacas Brahman durante el pre y postparto (19). Igualmente, una baja relación HDL:LDL ha sido un hallazgo observado en estudios previos con vacas Brahman subfértiles (3). La relación entre las lipoproteínas LDL y HDL puede verse afectada por desequilibrios de tipo nutricional, cuya evaluación no fue parte de los objetivos de este estudio. Se ha observado que la concentración de LDL es superior en bovinos cebú mestizos suplementados con semilla de algodón entera, aumentando el contenido de grasa en la ración (20).

La concentración de TG promedio en ambos sexos fue superior a la referencia (14). Similar a lo que sucede con el CT, la evaluación de la alimentación no fue un objetivo de este estudio, pero se ha señalado que la alimentación más que variables como el sexo o la edad pueden influir significativamente en los cambios observados en los metabolitos relacionados con el metabolismo lipídico (21).

Se ha descrito que una concentración de $\beta-\mathrm{OHB}$ menor a $0.46 \mathrm{mmol} / \mathrm{L}$ es considerada como valor referencia para bovinos (14). Los valores obtenidos en este estudio se encontraban ligeramente aumentados con respecto a este valor, pero no necesariamente compatibles con los observados para los casos de cetosis subclínica ( $>1.0 \mathrm{mmol} / \mathrm{L}$ ). Otros factores, no necesariamente relacionados con la dieta, pudieron haber influido para observar este leve aumento en la concentración de $\beta-\mathrm{OHB}$ (22).

La concentración de $\mathrm{T}_{4}$ en suero fueron similares a las encontradas en vacas no gestantes y no lactantes (23). Aunque en este estudio no se encontraron diferencias en $\mathrm{T}_{4}$ con respecto a la edad y sexo de los animales, éstos se describen como factores que pueden alterar la concentración de dicha hormona, además de otras situaciones como enfermedades, estrés, factores ambientales y composición racial de los animales $(24,25)$.

Las variables relacionadas con musculatura y cobertura grasa en el dorso y el anca no estaban relacionadas con los indicadores del metabolismo lipídico, excepto algunas correlaciones significativas y bajas que se observaron con la concentración de CT y lipoproteínas. Lo 
anterior estaría indicando que los metabolitos sanguíneos estudiados no guardan una relación lineal con la musculatura o la cobertura grasa, no siendo entonces indicadores útiles que permitan establecer un eventual desempeño productivo.

\section{Agradecimientos.}

A la Vicerrectoria de Investigaciones y Posgrados de la Universidad de Caldas y a la Asociación Colombiana de Criadores de Ganado Cebú Asocebú por el apoyo para desarrollar este estudio.

\section{REFERENCIAS}

1. Velásquez J, Alvarez L. Relación de medidas bovinométricas y de composición corporal in vivo con el peso de la canal en novillos Brahman en el Valle del Sinú. Revista Acta agronomica. [en linea]. 2004 [fecha de acceso: 9 de Junio de 2009]. Disponible en: http://www.revistas.unal. edu.co/index.php/acta_agronomica/article/ viewFile/100/216.

2. Chenoweth PJ. Bull sex drive and reproductive behavior. In: Topics in Bull Fertility. IVIS. [en linea]. 2000 [fecha de acceso: 21 Julio 2008]. Disponible en: http://www.ivis.org/ advances/Repro_Chenoweth/chenoweth/ chapter.asp?LA $=1$.

3. Villa NA, Pulgarín EF, Tabares PA, Angarita E, Ceballos A. Medidas corporales y concentración sérica y folicular de lípidos y glucosa en vacas Brahman fértiles y subfértiles. Pesq Agropec Bras. 2009;44:1198-204.

4. Guitou H. Interpretación y uso correcto de Ios DEP's como herramienta de selección. Unidad de Genética Animal, INTA Castelar [en linea]. 2004 [fecha de acceso: 11 de Junio de 2009]. Disponible en: http://www.produccion-animal.com.ar/ genetica_seleccion_cruzamientos/deps/60interpretacion_y_uso_deps.pdf.

5. Greiner SP, Rouse GH, Wilson DE, Cundiff $\mathrm{LV}$, Wheeler $\mathrm{TL}$. The relationship between ultrasound measurements and carcass fat thickness and longissimus muscle area in beef cattle. J Anim Sci. 2003;81:676-82.

6. Dohoo I, Wayne M, Stryhn H. Veterinary epidemiologic research. 2nd, editor. Charlottetown: AVC Inc; 2009.

7. Crews DH, Shannon $\mathrm{NH}$, Crews RE, Kemp RA. Weaning, yearling, and preharvest ultrasound measures of fat and muscle area in steers, bull, and heifers. J Anim Sci. 2002;80:2817-24.
8. Cerón MF, Montoya AE, Trujillo ER, Ramírez EJ, Monsalve ZI. Marcadores del gen leptina en bovinos cruzados con Angus, Cebú, Romosinuano y Blanco Orejinegro. Revista Científica FCV-LUZ 2009; XIX (4):371-81.

9. Vásquez R, Pulido J, Abuabara $Y$, Martínez R, Abadía B, Arreaza LC, et al. Patrones tecnológicos y calidad de la carne bovina en el Caribe Colombiano. Bogota: Produmedios; 2005.

10. Realini CE, Williams RE, Pringle TD, Bertrand JK. Gluteus medius and rump fat depths as additional live animal ultrasound measurements for predicting retail product and trimmable fat in beef carcass. J Anim Sci. $2001 ; 79: 1378-85$.

11. Bergen RD, Miller SP, Mandell IB, Robertson WM. Use of live ultrasound, weight and linear measurements to predict carcass composition of young beef bulls. Can J Anim Sci. 2005;85:23-35.

12. Guitou M, Sutz G, Baluk I. Interpretación y uso correcto de las diferencias esperadas entre progenie (DEP's) como herramienta de selección para la calidad de carne en Argentina. Segunda Parte. Rev Col Cien Pec. 2007;20:363-76.

13. Brethour JR. Using serial ultrasound measures to generate models of marbling and backfat thickness changes in feed lot cattle. J Anim Sci. 2000;78:2055-61.

14. Kaneko JJ, Harvey JW, Bruss ML. Clinical biochemistry of domestic animals. 6, editor. San Diego: Academic Press; 2008.

15. Coppo NB, Coppo JA, Lazarte MA. Intervalos de confianza para colesterol ligado a lipoproteínas de alta y baja densidad en suero de bovinos, equinos, porcinos y caninos. Rev Vet. 2003;14:3-10. 
16. Lammoglia MA, Willard ST, Hallford DM, Randel RD. Effects of dietary fat on follicular development and circulating concentrations of lipids, insulin, progesterone, estradiol-17 beta, 13,14-dihydro-15-keto-prostaglandin $\mathrm{F}$ ( 2 alpha), and growth hormone in estrous cyclic Brahman cows. J Anim Sci. 1997; 75:1591-600.

17. Cole NA, Brown MA, Phillips WA. Genetic $x$ environment interactions on blood constituents of Angus, Brahman, and reciprocal-cross cows and calves grazing common bermudagrass or endophyteinfected tall fescue. J Anim Sci. 2001;79:1151-61.

18. Coppo JA. Fisiología comparada del medio interno. 2nd, editor. Salta: Eucasa; 2008.

19. Villa NA, Osorio JM, Escobar DM, Ceballos A. Indicadores bioquímicos del balance energético en el periparto de vacas Brahman en pastoreo en el trópico Colombiano. Revista Científica FCV-LUZ. 2011;XXI(4):353-9.

20. Koza GA, Balbuena O, Mussart NB, Kucseva CD, Coppo JA. Cambios del lipidograma en novillos suplementados con semilla de algodón. Universidad Nacional del Nordeste. Comunicaciones científicas y tecnológicas. [en linea]. 2001 [fecha de acceso: 15 de julio de 2009]. Disponible en: http://www1.unne.edu.ar/cyt/2001/4Veterinarias/V-021.pdf.
21. Drackley JK. Lipid Metabolism. In: D'Mello JPF, editor. Farm animal metabolism and nutrition. Wallinford: CAB International; 2000. p. 97-120.

22. Agenas S, Heath MF, Nixon RM, Wilkinson JM, Phillips CJC. Indicators of under nutrition in cattle. Animal Welfeare. 2006;15(2):149-60.

23. Ashkar FA, Bartlewski PM, Singh J, Malhi PS, Yates KM, Singh T, et al. Thyroid hormone concentrations in systemic circulation and ovarian follicular fluid of cow. Exp Biol Med. 2010;235:215-21.

24. Matamoros R, Contreras PA, Wittwer F, Mayorga MI. Hipotiroidismo en rumiantes. Arch Med Vet. 2003;35(1):1-11.

25. Campos R, Giraldo L. Efecto de la raza y la edad sobre las concentraciones de hormonas tiroideas T3 y T4 de bovinos en condiciones tropicales. Acta Agronomica. 2008;57(2):137-41. 